\title{
Can radiotherapy potentiate the effectiveness of immune checkpoint inhibitors in lung cancer?
}

\author{
Monica Cheng ${ }^{1}$, Greg Durm², Nasser Hanna², Lawrence H Einhorn² \& Feng-Ming (Spring) \\ Kong*,1 \\ ${ }^{1}$ Department of Radiation Oncology, Indiana University School of Medicine, Indianapolis, IN 46202, USA \\ ${ }^{2}$ Department of Medicine, Division of Hematology/Oncology, Indiana University School of Medicine, Indianapolis, IN 46202, USA \\ * Author for correspondence: Tel.: +1 317944 2524; Fax: +1 317944 2486; fskong@iupui.edu
}

First draft submitted: 12 August 2017; Accepted for publication: 6 September 2017; Published online: 23 November 2017

Keywords: non-small-cell lung cancer $\bullet$ NSCLC • PD-1 immune checkpoint inhibition • radiation therapy • radioimmunotherapy $\bullet$ targeted therapy

Systemic therapy including platinum-based chemotherapy and targeted therapy has been a mainstay of treatment for the majority of patients with metastatic non-small-cell lung cancer (NSCLC). Recent breakthroughs in immune checkpoint blockade, along with a deeper understanding of tumor immune biology, have led to a paradigm shift in the standard of care for cancer treatment. By activating the immune system to recognize and eliminate cancer, immune checkpoint blockade has the potential to elicit durable responses and augment survival outcomes for a subset of patients [1,2]. In addition, the ability of radiotherapy to induce an immunogenic response and neutralize the immune-suppressive effects of the tumor microenvironment, uniquely positions it as a synergistic tool at the center of emerging multimodal therapies utilizing immune checkpoint blockade [3-7].

Immune checkpoint inhibitors of the PD-1/PD-L1 pathway have emerged as a promising new therapeutic strategy [8-11]. These agents have produced durable clinical responses and antitumor activity in Phase I trials, leading to subsequent randomized Phase II and III trials that demonstrated significant improvements in overall survival when compared with cytotoxic chemotherapy alone [12-16]. These studies have established PD-1/PD-L1 pathway blockade as a powerful new line of therapy for patients with advanced NSCLC following progression after platinum-based therapy [9,11]. Despite unprecedented improvements in clinical outcomes, less than $20 \%$ of patients respond to anti-PD-1/PD-L1 agents, necessitating strategies that explore combined treatment modalities in order to extend the benefits of immune checkpoint blockade to a broader patient population. The Phase II KEYNOTE-021 trial (NCT02039674), for example, evaluated the treatment of pembrolizumab in combination with pemetrexed and carboplatin and found significantly prolonged progression-free survival (median 13.0 vs 8.9 months; 6-month survival $92 \%$ for both arms) for patients with previously untreated metastatic nonsquamous NSCLC [17]. This trial, as well as the KEYNOTE-024 trial (NCT02142738), has served to bring PD-1 inhibition into the front-line setting for NSCLC [13].

Radiation has become known for its abscopal effect in lung cancer. There are many compelling reasons to combine radiotherapy with checkpoint inhibition in the treatment of NSCLC. Radiation affects the immune system in many ways including release of neoantigens, upregulation of PD-L1 on tumor cells, recruitment of T cells to the tumor bed and neutralization of the immune-suppressive effects of the tumor microenvironment. Over $60 \%$ of patients with NSCLC require radiotherapy at least once during their course of treatment, and radiotherapy used in combination with immune checkpoint blockade has shown potential in preclinical and early clinical studies [3,6,7,18]. A Phase II trial of consolidation pembrolizumab initiated 1-2 months after concurrent chemoradiation demonstrated safety of such treatment [19]. In fact, in May 2017, AstraZeneca and MedImmune announced positive results of the international Phase III PACIFIC trial (NCT 20125461), a double-blinded randomized trial to test the effect of immune checkpoint inhibitor, durvalumab in patients with stage III NSCLC who had not progressed following standard platinum-based chemotherapy and concurrent radiotherapy. In this trial, progression-free survival and overall survival were used as primary end points. On September 8, 2017, Antonia and colleagues reported an interim

Future $\because \cdots$ Medicine 
analysis of this Phase III PACIFIC study [20]. Among 713 lung cancer patients who underwent chemoradiotherapy and randomization, the median progression-free survival was 16.8 months with durvalumab versus 5.6 months with placebo $(\mathrm{p}<0.001)$. The median time to death or distant metastasis was substantially longer with durvalumab than with placebo (23.2 months vs 14.6 months; $\mathrm{p}<0.001)$ [20].

It is interesting to note that effect of radiation in patients treated under the Phase I KEYNOTE-001 trial (NCT01295827) published in Lancet Oncology in July 2017 which was to test established safety profile and antitumor activity of radiotherapy combined with pembrolizumab [21]. Among the 98 enrolled patients presenting with metastatic NSCLC, $43 \%$ received radiotherapy treatment for their NSCLC prior to the first cycle of pembrolizumab. For these patients, progression-free survival with pembrolizumab surpassed those who did not receive radiotherapy (median progression-free survival of 4.4 vs 2.1 months, 6-month progression-free survival of 49 vs $23 \%)$. Moreover, patients who received both radiotherapy and pembrolizumab treatment experienced improved overall survival compared with those who did not receive prior radiotherapy (median overall survival of $10.7 \mathrm{vs}$ 5.3 months, 6-month overall survival of 73 vs $45 \%$ ) [21]. These findings are reinforced by previous studies demonstrating the potential for radiotherapy combined with checkpoint blockade to improve local tumor control and in some cases exert an antitumor effect at sites distant from the irradiated field, a phenomenon called the abscopal effect $[6,7,22]$. By liberating tumor antigens and damage-associated molecular patterns through immunogenic cell death, radiotherapy can function as an 'in situ vaccine' that facilitates T-cell priming and may be used synergistically with PD-1 blockade to produce systemic antitumor effects [22,23].

With the development of multimodal therapies, it becomes increasingly critical to identify predictive biomarkers to guide patient selection for treatment [24,25]. The promise of biomarker-driven cancer treatment is best illustrated in the Phase II clinical trial reported by Le $e t$ al., which identified the predictive value of DNA mismatch repair (MMR) status, or microsatellite instability (MSI), for patients with metastatic colorectal cancer [26,27]. An MMRdeficient tumor predicted greater clinical response to treatment with pembrolizumab, leading to a significant improvement in progression-free survival [26,27]. In a subsequent study published in Science in July 2017, Le et al. further demonstrated the efficacy of PD-1 blockade across 12 types of tumors harboring MMR-deficiency, including lung cancer [28].

MMR-deficient cancers tend to harbor myriad somatic mutations, giving rise to mutation-associated neoantigens that sensitize tumors to immune checkpoint blockade [29]. By targeting this feature of cancer, immune checkpoint inhibition appears to elicit responses from tumors independent of the tissue of origin. On the basis of these promising results, the US FDA granted approval to pembrolizumab in May of 2017 for the treatment of patients with previously treated microsatellite instability-H or MMR-deficient solid tumors. This represents the first time that the FDA has approved a therapy completely independent of tumor site or histology and based solely on the presence of a specific biomarker. The immune modulating effect of radiotherapy will need to be taken into consideration in the management of various malignancies originating from different anatomic sites.

As immune checkpoint blockade continues to shape the landscape of NSCLC treatment, radiotherapy may have a promising role in synergistically enhancing the systemic response to immune checkpoint inhibition [30]. The identification of predictive biomarkers such as MMR-deficiency and mutation-associated neoantigens can identify responders to further improve the rates of response to immune checkpoint inhibitors and combining these agents with radiotherapy may further enhance their utility. By further unraveling the molecular underpinnings of radiation's immunomodulating effects, future and ongoing investigations have the potential to open new avenues in precision immunotherapy for the treatment of advanced NSCLC.

\section{Financial \& competing interests disclosure}

This work was supported in part by NIH/NCI grant R01CA142840 (PI: Kong). The authors have no other relevant affiliations or financial involvement with any organization or entity with a financial interest in or financial conflict with the subject matter or materials discussed in the manuscript apart from those disclosed.

No writing assistance was utilized in the production of this manuscript.

\section{References}

1. Boussiotis VA. Molecular and biochemical aspects of the PD-1 checkpoint pathway. N. Engl. J. Med. 375, 1767-1778 (2016).

2. Chen DS, Mellman I. Elements of cancer immunity and the cancer-immune set point. Nature 541, 321-330 (2017).

3. Kang J, Demaria S, Formenti S. Current clinical trials testing the combination of immunotherapy with radiotherapy. J. Immunother. Cancer 4, 51 (2016). 
4. Bernstein MB, Krishnan S, Hodge JW et al. Immunotherapy and stereotactic ablative radiotherapy (ISABR): a curative approach? Nat. Rev. Clin. Oncol. 13, 516-524 (2016).

5. Baumann M, Krause M, Overgaard J et al. Radiation oncology in the era of precision medicine. Nat. Rev. Cancer 16, 234-249 (2016).

6. Weichselbaum RR, Liang H, Deng L et al. Radiotherapy and immunotherapy: a beneficial liaison? Nat. Rev. Clin. Oncol. 14, 365-379 (2017).

7. Sharabi AB, Lim M, DeWeese TL et al. Radiation and checkpoint blockade immunotherapy: radiosensitisation and potential mechanisms of synergy. Lancet Oncol. 16, e498-e509 (2015).

8. Hirsch FR, Scagliotti GV, Mulshine JL et al. Lung cancer: current therapies and new targeted treatments. Lancet 389, $299-311$ (2017).

9. Soo RA, Stone ECA, Cummings KM et al. Scientific advances in thoracic oncology 2016. J. Thorac. Oncol. 12, 1183-1209 (2017).

10. Emens LA, Ascierto PA, Darcy PK et al. Cancer immunotherapy: opportunities and challenges in the rapidly evolving clinical landscape. Eur. J. Cancer 81, 116-129 (2017).

11. Hanna N, Johnson D, Temin S et al. Systemic therapy for stage IV non-small-cell lung cancer: American Society of Clinical Oncology clinical practice guideline update. J. Clin. Oncol. 35(30), 3484-3515 (2017).

12. Herbst RS, Baas P, Kim DW et al. Pembrolizumab versus docetaxel for previously treated, PD-L1-positive, advanced non-small-cell lung cancer (KEYNOTE-010): a randomised controlled trial. Lancet 387, 1540-1550 (2016).

13. Reck M, Rodriguez-Abreu D, Robinson AG et al. Pembrolizumab versus chemotherapy for PD-L1-positive non-small-cell lung cancer. N. Engl. J. Med. 375, 1823-1833 (2016).

14. Hellmann MD, Rizvi NA, Goldman JW et al. Nivolumab plus ipilimumab as first-line treatment for advanced non-small-cell lung cancer (CheckMate 012): results of an open-label, Phase 1, multicohort study. Lancet Oncol. 18, 31-41 (2017).

15. Rittmeyer A, Barlesi F, Waterkamp D et al. Atezolizumab versus docetaxel in patients with previously treated non-small-cell lung cancer (OAK): a Phase3, open-label, multicentre randomised controlled trial. Lancet 389, 255-265 (2017).

16. Peters S, Gettinger S, Johnson ML et al. Phase II trial of atezolizumab as first-line or subsequent therapy for patients with Programmed Death-Ligand 1-selected advanced non-small-cell lung cancer (BIRCH). J. Clin. Oncol. 35, 2781-2789 (2017).

17. Langer CJ, Gadgeel SM, Borghaei $\mathrm{H}$ et al. Carboplatin and pemetrexed with or without pembrolizumab for advanced, non-squamous non-small-cell lung cancer: a randomised, Phase 2 cohort of the open-label KEYNOTE-021 study. Lancet Oncol. 17, 1497-1508 (2016).

18. Shabason JE, Minn AJ. Radiation and Immune checkpoint blockade: from bench to clinic. Semin. Radiat. Oncol. 27, $289-298$ (2017).

19. Durm G, Johnson C, Jalal S et al. Safety and feasibility of consolidation pembrolizumab following concurrent chemoradiation for unresectable stage III non-small cell lung cancer: hoosier cancer research network LUN14-179. J. Clin Oncol. Suppl. Abstract 8523 (2017).

20. Antonia SJ, Villegas A, Daniel D et al. Durvalumab after chemoradiotherapy in stage III non-small-cell lung cancer. N. Engl. J. Med. doi:10.1056/NEJMoa1709937 (2017) (Epub ahead of print).

21. Shaverdian N, Lisberg AE, Bornazyan K et al. Previous radiotherapy and the clinical activity and toxicity of pembrolizumab in the treatment of non-small-cell lung cancer: a secondary analysis of the KEYNOTE-001 Phase 1 trial. Lancet Oncol. 18, 895-903 (2017).

22. Demaria S, Coleman CN, Formenti SC. Radiotherapy: changing the game in immunotherapy. Trends Cancer 2, $286-294$ (2016).

23. Wennerberg E, Lhuillier C, Vanpouille-Box C et al. Barriers to radiation-induced in situ tumor vaccination. Front. Immunol. 8, 229 (2017).

24. Lesterhuis WJ, Bosco A, Millward MJ et al. Dynamic versus static biomarkers in cancer immune checkpoint blockade: unravelling complexity. Nat. Rev. Drug Discov. 16, 264-272 (2017).

25. Gulley JL, Berzofsky JA, Butler MO et al. Immunotherapy biomarkers 2016: overcoming the barriers. J. Immunother. Cancer 5, 29 (2017).

26. Le DT, Uram JN, Wang H et al. PD-1 blockade in tumors with mismatch-repair deficiency. N. Engl. J. Med. 372, $2509-2520$ (2015).

27. Llosa NJ, Cruise M, Tam A et al. The vigorous immune microenvironment of microsatellite instable colon cancer is balanced by multiple counter-inhibitory checkpoints. Cancer Discov. 5, 43-51 (2015).

28. Le DT, Durham JN, Smith KN et al. Mismatch-repair deficiency predicts response of solid tumors to PD-1 blockade. Science 357, 409-413 (2017).

29. Yarchoan M, Johnson BA 3rd, Lutz ER et al. Targeting neoantigens to augment antitumour immunity. Nat. Rev. Cancer 17, 209-222 (2017).

30. Mouw KW, Goldberg MS, Konstantinopoulos PA et al. DNA damage and repair biomarkers of immunotherapy response. Cancer Discov. 7, 675-693 (2017). 
